\title{
Effortlessly transfer and back up data
}

Dentsply Sirona Hub offers easy working and secure handling of CAD/CAM data. This device saves CEREC data and allows other users in the practice network to access it. In addition to saving case data locally on a CEREC acquisition unit, they are stored in the Hub, where two copies are saved. Other acquisition units or computers that are equipped with CEREC or CEREC Premium Software can access the data from anywhere in the practice network. It is such an easy and natural process that you would think the data were saved directly on that particular workstation.

Hub also synchronises patient data with Sidexis. For example, if a patient is scanned with CEREC Omnicam and their personal data have already been stored for an X-ray, there is no need to enter the information again on the acquisition unit.

There is also no need for USB thumb drives or shared network folders with Dentsply Sirona Hub: the Hub connection enables CEREC data to be opened and processed from any $\mathrm{PC}$ in the practice network with CEREC or CEREC Premium Software - to design restorations, for example. This offers the advantage that the digital model is saved on Hub in real time once the dentist has completed a scan. What's more, Hub independently keeps a task list of all the cases that have not yet been completed and notifies their status. The technician or dentist can open

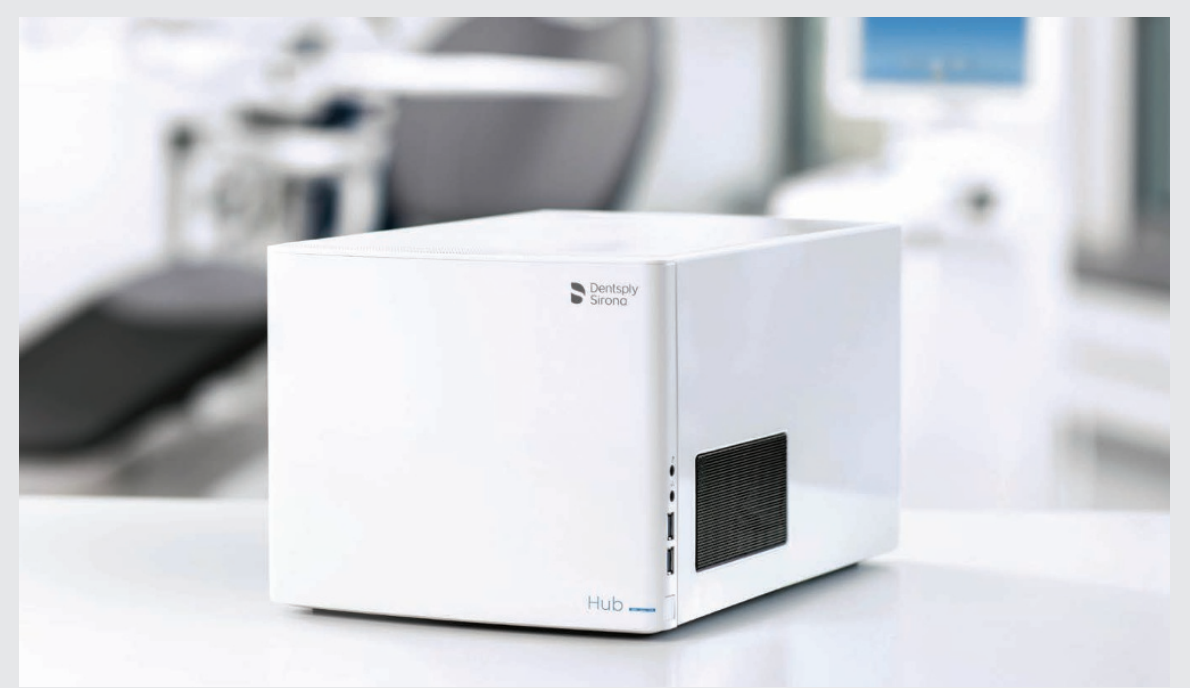

the scan from the list on another computer and process it, leaving the CEREC unit in the operatory free to take another scan. This gives clinicians considerably more freedom when organising the workflows in their practice and makes them more efficient.

Hub is intended to assume the future role as the practice's data centre. Consequently, its functionality will expand gradually and be available to users through free updates. The functions that can be used at market launch will benefit users with multiple acquisition units or practice laboratories in particular. Users no longer need to import and export data manually, and can now be absolutely certain that data are transferred and saved with strong encryption, something that is not possible with conventional solutions. This system allows practices to satisfy the highest requirements of data security. Hub Installation couldn't be simpler - just connect it to the power source and network, and switch it on. Its 'Plug \& Play' functionality means that Hub does the rest automatically, and it connects independently to all compatible devices and workstations in the network.

Hub was presented for the first time at Dentsply Sirona World 2017 in Las Vegas. Expansions for the device are planned for the future to make Hub a centre of communication in the practice. Great for early buyers: all future updates for Hub are free of charge.

www.dentsplysirona.com.

\section{Smart toothbrush will provide real time guidance to patients}

On 1 November 2017 at an exclusive event in Central London, a revolutionary new dental product was launched to tackle Britain's poor toothbrushing habits. Brushlink is a device that costs less than $£ 30$ that will transform any toothbrush into a smart toothbrush.

Brushlink combines a tracking device attached to your toothbrush with a smartphone app. The smartphone app provides the user with real time brushing guidance and performance monitoring. Each brushing session is scored and monitored and the cumulative data can be shared with dental professionals so that they can provide targeted help and advice to the patient.

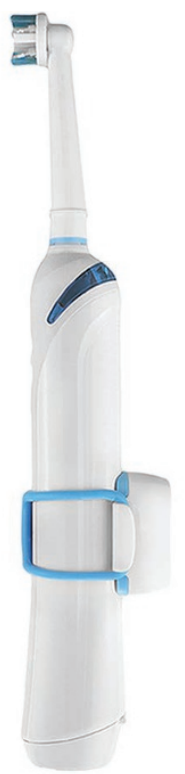

The tracking device is a tiny hi-tech Bluetooth unit that attaches easily to any toothbrush and monitors brushing frequency, duration and, for the first time, angulation, so that correct brushing angles can be encouraged, reinforced and monitored to help prevent gum disease.

Brushlink was founded by CEO Dr Dev Patel (featured in the $B D J$ earlier this month: http://go.nature.com/2hoKg8X).

Through using Brushlink, patients can sync their brushing data with a Brushlink certified dental practice, for tailored dental hygiene recommendations, and earn bespoke discounts.

For more information about Brushlink and to order, visit https://brushlink.com/. 